Article

\title{
Spatial Coherence of Backscattered Signals in Multi-Line Transmit Ultrasound Imaging and Its Effect on Short-Lag Filtered-Delay Multiply and Sum Beamforming
}

\author{
Giulia Matrone ${ }^{1,2, *}$ and Alessandro Ramalli ${ }^{3,4}$ (iD) \\ 1 Department of Electrical, Computer and Biomedical Engineering, University of Pavia, 27100 Pavia, Italy \\ Centre for Health Technologies, University of Pavia, 27100 Pavia, Italy \\ 3 Laboratory on Cardiovascular Imaging and Dynamics, Department of Cardiovascular Sciences, KU Leuven, \\ 3000 Leuven, Belgium; alessandro.ramalli@kuleuven.be \\ 4 Department of Information Engineering, University of Florence, 50139 Florence, Italy \\ * Correspondence: giulia.matrone@unipv.it; Tel.: +39-0382-985918
}

Received: 6 February 2018; Accepted: 21 March 2018; Published: 23 March 2018

\begin{abstract}
In Multi-Line Transmission (MLT), high frame-rate ultrasound imaging is achieved by the simultaneous transmission of multiple focused beams along different directions, which unfortunately generates unwanted artifacts in the image due to inter-beam crosstalk. The Filtered-Delay Multiply and Sum (F-DMAS) beamformer, a non-linear spatial-coherence (SC)-based algorithm, was demonstrated to successfully reduce such artifacts, improving the spatial resolution at the same time. In this paper, we aim to provide further insights on the working principle and performance of F-DMAS beamforming in MLT imaging. First, we carry out an analytical study to analyze the behavior and trend of backscattered signals SC in MLT images, when the number of simultaneously transmitted beams and/or their angular spacing change. We then reconsider the F-DMAS algorithm proposing the "short-lag F-DMAS" formulation, in order to limit the maximum lag of signals used for the SC computation on which the beamformer is based. Therefore, we investigate in simulations how the performance of short-lag F-DMAS varies along with the maximum lag in the different MLT configurations considered. Finally, we establish a relation between the obtained results and the signals SC trend.
\end{abstract}

Keywords: filtered-delay multiply and sum beamforming; multi-line transmission; spatial coherence; ultrasound imaging

\section{Introduction}

Spatial coherence (SC) of ultrasound backscattered echoes has been the object of numerous works in the ultrasound imaging field. The first works date back to the 90s [1-5], when it was proposed to extend to pulse-echo ultrasound the Van Cittert Zernike (VCZ) theorem of statistical optics, which describes the spatial covariance of the wave field generated by an incoherent source [1].

Recently, the attention has been focused towards exploiting the concept of spatial coherence for the development of new image reconstruction and beamforming techniques. Some of the most renowned coherence-based methods are coherence-factor [6], phase-coherence and sign-coherence [7] beamforming, Filtered-Delay Multiply and Sum (F-DMAS) beamforming [8], and Short-Lag Spatial Coherence (SLSC) imaging [9]. The latter is a technique which directly generates an image of the spatial coherence of backscattered echoes evaluated at short lags, while all other methods aim at generating B-mode images, either via a coherence-based weighting or through non-linear 
correlation-like operations as in F-DMAS. Anyway, all these techniques use some estimate of spatial coherence to increase image quality. In general, they show enhanced clutter rejection and contrast improvement capabilities [9,10], which often also come along with an increase of lateral resolution $[7,8]$. For these reasons, in several studies, they have been proposed for use in application to techniques that partially sacrifice some of the above-mentioned image quality metrics to improve other crucial factors in ultrasound medical imaging, such as the frame rate. This is the case of well-known techniques like plane-wave imaging [11,12], multi-line acquisition (also called parallel beamforming) [13], and multi-line transmission (MLT) [14].

MLT, as the name suggests, is based on the use of multiple beams, which are transmitted simultaneously in the medium [15], reducing the acquisition time by a factor equal to the number of transmitted beams. It has recently gained a lot of interest, especially for application in cardiac ultrasound imaging [15], where the possibility to observe and track heart motion in real-time is of the uttermost importance for diagnostic purposes. In [15], it has been shown that, by applying a Tukey apodization window on both transmit and receive sides, improved performance can be achieved thanks to the lowering of cross-talk artifacts that arise when multiple beams are transmitted simultaneously, but at the expense of lateral resolution. Other works in the literature have addressed this same problem, such as [16-18].

Our group has recently worked towards the joint use of MLT with a new non-linear beamforming algorithm [19], i.e., F-DMAS [8,20,21], and showed how F-DMAS overcomes the main problems that have so far limited the use of MLT in clinical practice (i.e., cross-talk artifacts and low lateral resolution). This algorithm basically consists of computing the aperture spatial autocorrelation in reception; however, the pulse-echo response is heavily jeopardized by MLT imaging [22], hence the question we address in this work is: does this affect spatial coherence? If so, how?

To understand how spatial coherence varies in different imaging setups and with different image acquisition strategies can indeed be very useful, in that it allows us to explain the achievable performance and possible effects that could be observed in images generated with the previously mentioned spatial-coherence-based beamformers, which are obviously influenced by its behavior. In particular, here we are interested in analyzing the performance of F-DMAS when combined with different MLT strategies and when the spatial correlation operation, on which this algorithm is based, is only computed between signals within a certain maximum lag.

Therefore, the work presented here is organized in two parts. In the first part, an analytical study of the spatial coherence trend in a simulated uniform medium with MLT imaging is presented. Such analysis is performed by varying the main MLT parameters, i.e., the number of transmitted beams and/or their angular separation. In the second part, a modified version of F-DMAS beamforming, called short-lag F-DMAS, is introduced; the simulated results of MLT imaging with short-lag F-DMAS beamforming are analyzed in the different MLT scenarios mentioned above, also studying how the maximum spatial lag between signals that enter this beamformer influences image quality.

In the next sections, we will briefly recall the theoretical background of spatial coherence in ultrasound imaging and the main steps of the F-DMAS algorithm; the simulation setup will be described and the strategy used to study the trend of coherence with MLT will be presented (Section 2). Finally, the results of the coherence analytical study and F-DMAS performance in the considered test cases will be shown and discussed (Section 3). Conclusions will be provided in Section 4.

\section{Materials and Methods}

In Section 2.1, the spatial correlation concept is introduced and mathematically described, followed by a presentation of the MLT technique in Section 2.2. Subsequently, a description of short-lag F-DMAS beamforming is provided in Section 2.3. The simulation setup and the whole study organization are illustrated in Section 2.4. 


\subsection{Spatial Coherence}

Spatial coherence (also called spatial correlation) is a measure of the coherence of the ultrasound beam reflected by a diffuse scattering medium. The VCZ theorem [2] states that spatial coherence can be obtained by computing the Fourier transform of the field intensity. Hence, in a classic B-mode scan, where dynamic focusing is only applied in reception, spatial coherence reaches its maximum value at the transmit focus, where the beam is narrower; moreover, the coherence function has a triangular shape, being the field a $\operatorname{sinc}^{2}$ for a uniformly weighted aperture. Away from the focus, the beam widens and spatial coherence decreases. The same effect is observed in all those cases in which a decorrelation occurs, e.g., because of the presence of acoustic and/or electronics noise, beam sidelobes, and aberrations, etc. $[9,20,23]$.

For each discrete time sample $t$, we define the normalized spatial covariance as:

$$
C_{N}(l, t)=\frac{C(l, t)}{C(0, t)}=\frac{\sum_{n=1}^{N-l} \sum_{t=t_{1}}^{t_{2}} s_{n}(t) s_{n+l}(t)}{\sum_{n=1}^{N} \sum_{t=t_{1}}^{t_{2}} s_{n}^{2}(t)}
$$

where $s_{n}(t)$ are the backscattered radio-frequency (RF) signals received by each $n$-th transducer of the $N$-element receive aperture and focused (delayed). $l$ is an integer number representing the spatial lag, i.e., the number of elements between the couple of multiplied signals $s_{n}(t)$ and $s_{n+l}(t)(l=0 \ldots N-1)$, whose product is integrated over a short time interval $t=\left[t_{1} ; t_{2}\right] . C(l, t)$ is the spatial covariance at the $l$-th lag; the normalization factor (denominator of Equation (1)) is given by the zero-lag covariance $C(0, t)$, as in [3].

Thus, a metric which accounts for the total spatial coherence $S C(t)$ (similar to what is proposed in [10] for SLSC imaging) can be computed as:

$$
S C(t)=\sum_{l=0}^{N-1} C_{N}(l, t)
$$

\subsection{Multi-Line Transmission}

MLT consists of transmitting $N_{T X}$ beams simultaneously in different focusing directions. After each transmission (TX), the backscattered signals are collected by the array elements in reception (RX) and beamformed in parallel along the considered steering directions. Then, the beams are moved by an angular step $\theta_{S T E P}=\theta_{S E C T} / N_{L I N E S}$ in order to cover the full $\theta_{S E C T}$-wide image field of view with $N_{\text {LINES }}$ scan lines. Therefore, the simultaneous TX beams are separated by an angular distance $\theta_{T X}=\theta_{S E C T} / N_{T X}[22]$.

To achieve multiple beam TX, a set of focusing delays has to be computed for each of the simultaneous TX focusing directions. Thus, the MLT excitation pulse is obtained by summing up the excitation signals that would be used to focus the TX beam along each one of the considered steering directions in the classic single-line transmission (SLT) case.

As explained in [22], the main problem with MLT is the presence, in the final image, of the so called cross-talk artifacts due to interferences between the multiple beams.

To better understand how such artifacts generate, we have to recall the array theory [24], according to which the TX $\left(h_{T X}\right)$ and $\mathrm{RX}\left(h_{R X}\right)$ ultrasound beams at the focal depth can be generally expressed as follows:

$$
\begin{aligned}
& h_{T X}(u)=\operatorname{sinc}\left(\frac{p_{x} u}{\lambda}\right) \operatorname{sinc}\left[\frac{N p_{x}}{\lambda}\left(u-\sum_{j=-\infty}^{+\infty} \frac{j \lambda}{p_{x}}-u^{T X}\right)\right] \\
& h_{R X}(u)=\operatorname{sinc}\left(\frac{p_{x} u}{\lambda}\right) \operatorname{sinc}\left[\frac{N p_{x}}{\lambda}\left(u-\sum_{j=-\infty}^{+\infty} \frac{j \lambda}{p_{x}}-u^{R X}\right)\right]
\end{aligned}
$$


where $u=\sin (\theta), u^{T X}$ refers to the focusing direction of the TX beam $\left(\theta^{T X}\right)$ and $u^{R X}$ to the focusing direction of the RX beam $\left(\theta^{R X}\right)$. $N$ is the number of array elements, $p_{x}$ is the array pitch, and $\lambda$ is the wavelength. Then, in the classic SLT case (i.e., each time one beam is transmitted and received), the pulse-echo $\left(h_{P E}\right)$ response is given by:

$$
h_{P E}(u)=h_{T X}(u) h_{R X}(u) .
$$

In MLT imaging, instead, several beams are transmitted in parallel, thus we have a summation in the TX beam expression:

$$
h_{T X}(u)=\operatorname{sinc}\left(\frac{p_{x} u}{\lambda}\right) \sum_{i=1}^{N_{T X}} \operatorname{sinc}\left[\frac{N p_{x}}{\lambda}\left(u-\sum_{j=-\infty}^{+\infty} \frac{j \lambda}{p_{x}}-u_{i}^{T X}\right)\right]
$$

where $u_{i}^{T X}$ refers to the focusing direction of the $i$-th TX beam $\left(\theta_{i}^{T X}\right)$. Hence, in this case the pulse-echo beam in (5) is made of $N_{T X}$ terms, where one term is the one generated when $\theta_{i}^{T X}=\theta^{R X}$, i.e., the $T X$ and RX responses are equal (as in a classic SLT scan), and the other terms with $\theta_{i}^{T X} \neq \theta^{R X}$ are the so called cross-talk contributions that originate from interferences among the TX and RX beams. Consequently, in the SLT case, when $N_{T X}=1$, the pulse-echo beam has a sinc ${ }^{2}$ shape, while in MLT with $N_{T X}>1$, the beam has a more complex shape, with $N_{T X}$ peaks in the simultaneous TX focusing directions, as shown in Figure 1 for MLT with four or 12 TX beams (i.e., 4-MLT and 12-MLT).
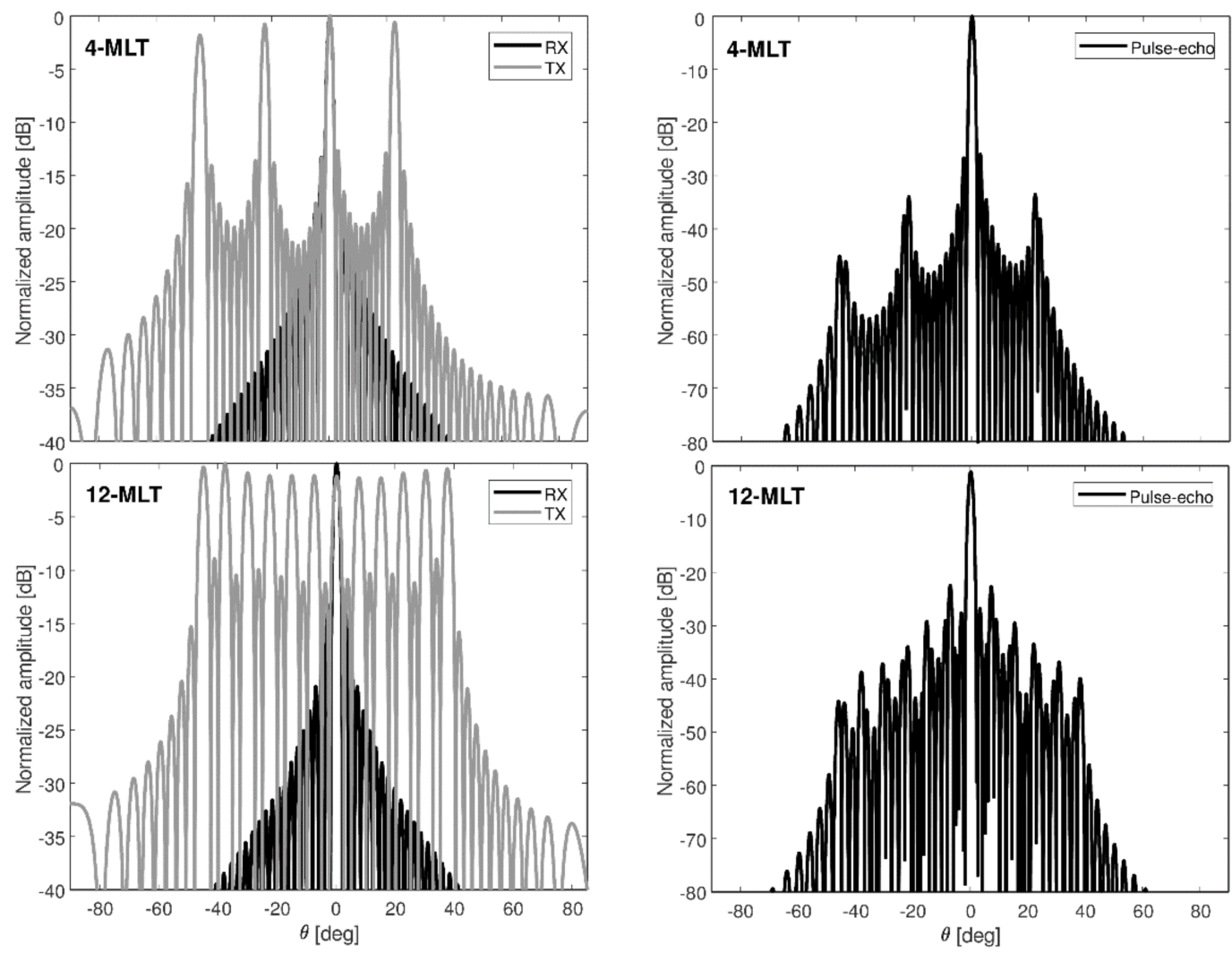

Figure 1. Examples of theoretical beampatterns in 4-MLT and 12-MLT: in the panels on the left, the TX (gray) and RX (black) beampatterns are plotted in each MLT case, when the RX beam is steered in one of the TX beams' direction (e.g., at $0^{\circ}$ ); on the right, the resulting pulse-echo beam shapes are shown.

In particular, interferences generate two types of cross-talk, i.e., the so called TX cross-talk, caused by interferences among TX beam side lobes and the RX beam main lobe, and RX cross-talk, caused by 
interferences between the TX beam main lobe and RX beam side lobes [22], which appear as artifacts around the beam main lobe along the axial or lateral direction, respectively.

\subsection{Short-Lag Filtered-Delay Multiply and Sum Beamforming}

F-DMAS beamforming derives from microwave imaging [25] and was recently introduced in ultrasound B-mode imaging by some of the authors [8]. This algorithm is based on the computation of the RX aperture spatial autocorrelation function. In F-DMAS, after focusing, the received RF signals $s_{n}(t)$ are amplitude-rescaled, by means of the signed square-root operator, and then combinatorially coupled and multiplied. The beamformed output is thus computed as:

$$
y_{D M A S}(t)=\sum_{n=1}^{N-1} \sum_{m=n+1}^{N} \operatorname{sign}\left(s_{n}(t) s_{m}(t)\right) \cdot \sqrt{\left|s_{n}(t) s_{m}(t)\right|}
$$

$y_{F D M A S}(t)$ is subsequently obtained by band-pass (BP) filtering $y_{D M A S}(t)$, in order to pass the second-harmonics that originates after signal cross-multiplications, and to attenuate as much as possible the baseband and higher frequency components which originate from such non-linear operations. After beamforming, the obtained RF image lines are demodulated using the Hilbert transform, normalized, and logarithmically compressed to produce the final B-mode image.

The F-DMAS formulation in (7) includes in the summation all possible couples among received RF signals, whose spatial lag $l=m-n$ varies from a minimum of 1 to a maximum $M$ equal to $N-1$ (i.e., only auto-product terms are excluded). Since we are interested in studying the relation between SC and F-DMAS in MLT imaging, in this work we present the short-lag F-DMAS formulation, which limits the maximum lag $M$ considered in the F-DMAS cross-multiplication stage. In particular, we will analyze the quality of images generated by using the following short-lag F-DMAS formulation:

$$
y_{D M A S}(t, M)=\sum_{l=1}^{M} \sum_{n=1}^{N-l} \operatorname{sign}\left(s_{n}(t) s_{n+l}(t)\right) \cdot \sqrt{\left|s_{n}(t) s_{n+l}(t)\right|},
$$

with $M=1,2,3, \ldots, N-1$.

\subsection{Simulation Setup and Study Organization}

In this work, Matlab (The MathWorks, Natick, MA, USA) simulations with Field II $[26,27]$ were carried out by modeling a 64-element phased array probe with a $340 \mu \mathrm{m}$ pitch. The central working frequency was $2 \mathrm{MHz}$, and a 2-cycle Hanning-weighted sinusoidal burst was used as the excitation signal. The transmit focus was set at a $70 \mathrm{~mm}$ depth and dynamic focusing was implemented in reception. A $100 \mathrm{MHz}$ sampling frequency was considered.

First, the analytical study on the spatial coherence trend was performed by simulating a numerical uniform speckle phantom, made of 140,000 scattering points with a Gaussian amplitude distribution, randomly placed in a $100 \times 1 \times 70 \mathrm{~mm}^{3}$ volume centered around $(x, y, z)=(0,0,65) \mathrm{mm}$ (i.e., $>10$ scatterers per resolution cell).

In B-mode imaging with standard dynamic RX focusing, the spatial coherence is known to decrease before and after the fixed TX focus [20,23]; thus, in this work, both the normalized covariance $C_{N}(l, t)$ and total spatial correlation $S C(t)$ were evaluated using Equations (1) and (2), and averaging the values obtained in a small $5 \times 5 \mathrm{~mm}^{2}$ area centered at $(x, z)=(0,70) \mathrm{mm}$, i.e., over the TX focus. Three different scenarios were analyzed (Table 1):

1. the number of MLT beams varies (i.e., $N_{T X}=1 / 4 / 6 / 8 / 12$ ), but the total image sector is fixed $\left(\theta_{S E C T}=90^{\circ}\right)$, as well as the number of lines (192); consequently, the angle among the TX beams $\left(\theta_{T X}=\theta_{S E C T} / N_{T X}\right)$ changes together with the number of beams (usually, this is the classic MLT implementation); 
2. the number of MLT beams varies (i.e., $\left.N_{T X}=1 / 4 / 6 / 8 / 12\right)$, but the same angle $\left(\theta_{T X}\right)$ among the beams is used in all configurations; in particular, this angle was set to be equal to the one that would be obtained applying 12 -MLT to scan a $90^{\circ}$ sector (i.e., $\theta_{12}$ ). Thus, in this case, the total image sector also changes in the different MLT configurations;

3. the number of beams is fixed (i.e., $N_{T X}=4$ ), while the angle among them changes, as it would do in $4 / 6 / 8 / 12$-MLT when a $90^{\circ}$ sector is acquired. Thus, also here, the total image sector changes in each case.

Table 1. MLT configurations: number of TX beams and angular spacing.

\begin{tabular}{cccccc}
\hline & Scenario 1 & \multicolumn{2}{c}{ Scenario 2 } & \multicolumn{2}{c}{ Scenario 3 } \\
\hline $\boldsymbol{N}_{\boldsymbol{T X}}$ & $\boldsymbol{\theta}_{\boldsymbol{T X}}$ & $\boldsymbol{N}_{\boldsymbol{T X}}$ & $\boldsymbol{\theta}_{\boldsymbol{T X}}$ & $\boldsymbol{N}_{\boldsymbol{T X}}$ & $\boldsymbol{\theta}_{\boldsymbol{T X}}$ \\
\hline 1 & $\theta_{1}=90^{\circ}$ & 1 & $\theta_{12}$ & - & - \\
4 & $\theta_{4}=\theta_{1} / 4$ & 4 & $\theta_{12}$ & 4 & $\theta_{4}$ \\
6 & $\theta_{6}=\theta_{1} / 6$ & 6 & $\theta_{12}$ & 4 & $\theta_{6}$ \\
8 & $\theta_{8}=\theta_{1} / 8$ & 8 & $\theta_{12}$ & 4 & $\theta_{8}$ \\
12 & $\theta_{12}=\theta_{1} / 12$ & 12 & $\theta_{12}$ & 4 & $\theta_{12}$ \\
\hline
\end{tabular}

These three scenarios were specifically chosen to evaluate the influence of the number of simultaneously transmitted beams or of their angular spacing, or of both factors, on the SC trend.

The second part of this study aimed at testing the performance of short-lag F-DMAS beamforming in MLT imaging. Results were also compared to those of DAS with Tukey apodization in RX. In both cases, Tukey apodization was also applied in TX, in order to reduce TX cross-talk [22].

Image quality was evaluated in terms of lateral resolution (at $-6 \mathrm{~dB}$ ), contrast ratio (CR), contrast-to-noise ratio (CNR), and speckle signal-to-noise ratio (sSNR) [8]:

$$
\begin{gathered}
C R=20 \log _{10}\left(\frac{\mu_{c y s t}}{\mu_{b c k}}\right), \\
C N R=\frac{\left|\mu_{c y s t}-\mu_{b c k}\right|}{\sqrt{\sigma_{c y s t}^{2}+\sigma_{b c k t}^{2}}}, \\
s S N R=\frac{\mu_{b c k}}{\sigma_{b c k}} .
\end{gathered}
$$

Point spread function (PSF) simulations were carried out considering a series of vertically aligned point scatterers along the $x=0 \mathrm{~mm}$ axis, from $z=10 \mathrm{~mm}$ to $90 \mathrm{~mm}$ with a $20 \mathrm{~mm}$ step; in addition, resolution measurements were made at the focal depth on the PSF at $(x, z)=(0,70) \mathrm{mm}$. CR, CNR, and SSNR were instead evaluated by simulating a numerical phantom of 140,000 points (i.e., $>10$ scatterers per resolution cell), with a $10-\mathrm{mm}$-diameter anechoic cyst centered at $(x, z)=(0,70) \mathrm{mm}$, in a $100 \times 1 \times 70 \mathrm{~mm}^{3}$ uniform tissue background starting at $z=30 \mathrm{~mm}$. Measurements were performed considering two 6-mm-diameter circular areas inside and outside the cyst.

\section{Results and Discussion}

\subsection{Spatial Coherence Trends in MLT Images of a Homogeneous Phantom}

Figure 2 shows the trend of backscattered signals SC (averaged over a small region around the focal depth, as said before), measured on the simulated uniform phantom images. The trend is plotted for SLT and 4/6/8/12 MLT in the three scenarios described in Section 2.4. Figure 3 represents the total spatial coherence values obtained by computing a summation over lags of the values plotted in Figure 2, for each MLT configuration (i.e., for each curve). 
Figure 2 shows that SC has a triangular trend in SLT, which decreases from 1 at lag 0 to about -0.1 at lag 63 . This trend is consistent with the one predicted by the VCZ theorem, which states that (at the focal depth) the SC of backscattered signals is the Fourier transform of the field intensity.

By looking at Figure 2a, we can also see that, when the number of TX beams increases (decreasing at the same time the angle among them), SC decreases more rapidly in the short-lag region, immediately after the peak at lag 0 , and varies following a sort of damped oscillation for each different MLT case. On the whole, as shown by Figure 3a, total coherence decreases non-linearly as the number of MLT beams increases.
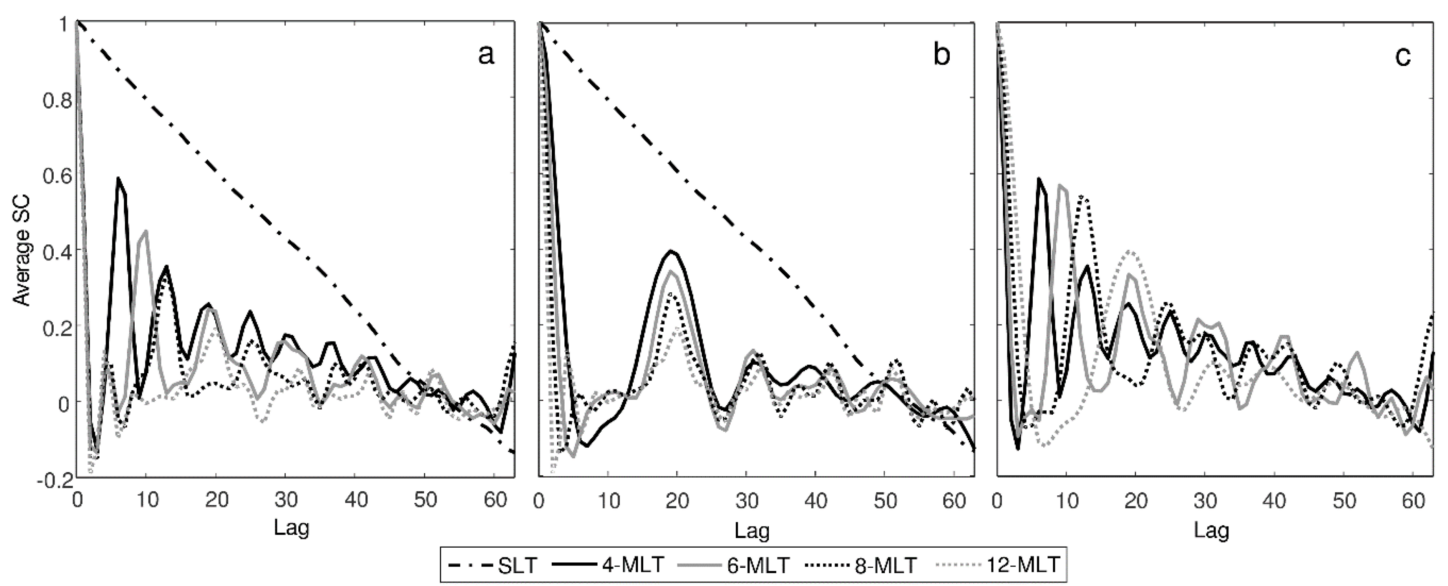

Figure 2. Normalized spatial covariance trend vs. lags, measured on the simulated uniform phantom data, and averaged over a $5 \times 5 \mathrm{~mm}^{2}$ area around $(x, z)=(0,70) \mathrm{mm}$ in the different scenarios of Table 1 : (a) both the number of TX beams and the angle among them varies (as in SLT or 4/6/8/12-MLT); (b) only the number of TX beams varies $\left(N_{T X}=1 / 4 / 6 / 8 / 12\right)$, while the angular distance among them is fixed to the one of 12-MLT; (c) the number of TX beams is set to four, while the angular distance among them is the one of 4/6/8/12-MLT (in this last case, the legend refers to the number of TX beams which would determine the angular distance).
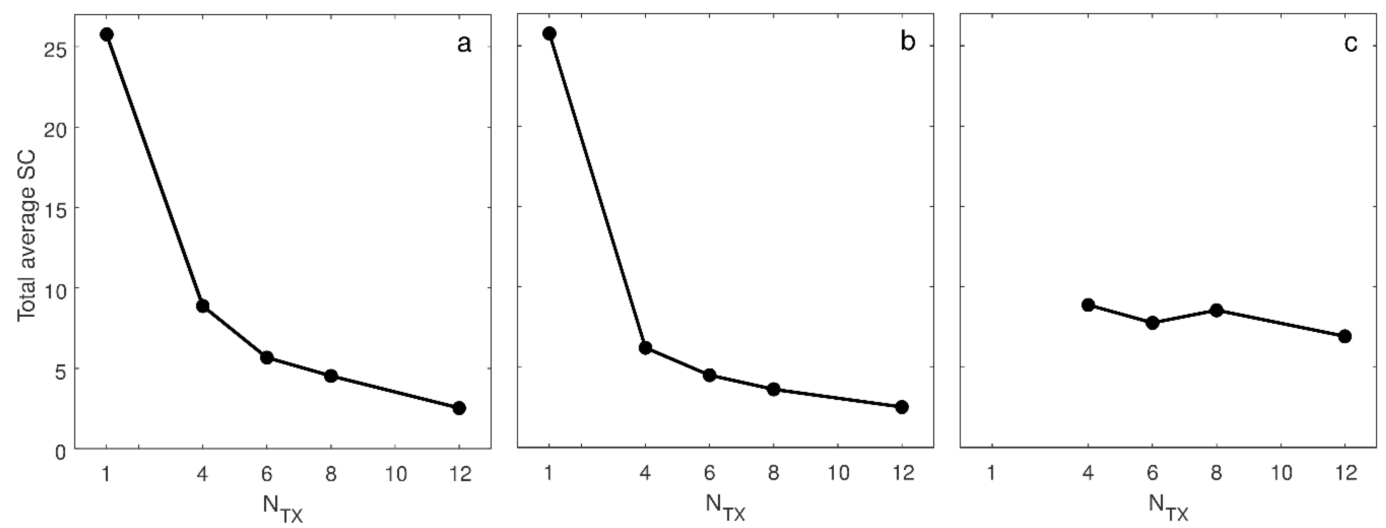

Figure 3. Total average spatial coherence, measured in a $5 \times 5 \mathrm{~mm}^{2}$ area around $(x, z)=(0,70) \mathrm{mm}$ in the different scenarios of Table 1: (a) both the number of TX beams and the angle among them varies (as in SLT or 4/6/8/12-MLT); (b) only the number of TX beams varies $\left(N_{T X}=1 / 4 / 6 / 8 / 12\right)$, while the angular distance among them is fixed to the one of 12-MLT; (c) the number of TX beams is set to four, while the angular distance among them is the one of 4/6/8/12-MLT (in this last case, the legend refers to the number of TX beams which would determine the angular distance).

In order to understand which factor determines such trends and an overall decrease of SC, we can look at Figure $2 b, c$ and Figure $3 b$,c. Figure $2 b$ refers to the case in which only the number of 
simultaneous TX beams varies, but the angle among them is fixed; in this way, it is possible to analyze the dependence of the SC trend on $N_{T X}$ only. The plots clearly show that this time the SC oscillatory trend becomes similar for all MLT configurations (except for SLT), but the lobes have different peak amplitudes and widths, which still makes the total SC decrease when increasing the number of TX beams (Figure $3 b$ ). On the other hand, when the number of MLT beams $N_{T X}$ is fixed, while the angle among them varies, we see in Figure $2 \mathrm{c}$ that the SC trend shows a different pitch between secondary lobes. Instead, total SC becomes similar in all cases (Figure 3c).

Therefore, by merging the information derived from the plots in Figures 2 and 3, we can observe that in all cases SC decreases when the lag between backscattered signals increases; this decreasing trend only follows a triangular pattern in the SLT case, when the pulse-echo beam is approximately a $\operatorname{sinc}^{2}$, while a sort of damped oscillatory behavior is shown in MLT imaging, as expected because of the more complex beam shape obtained when multiple beams are transmitted simultaneously (cf. Figure 1). Furthermore, Figure $2 b, c$ and Figure $3 b, c$ demonstrate that it is the angular distance between the multiple TX beams (i.e., $\theta_{T X}$ ) that mainly influences the SC trend over lags in MLT imaging, while the total SC value depends on the number of TX beams. When $N_{T X}$ is fixed to four, for example, as in Figures $2 \mathrm{c}$ and $3 \mathrm{c}$, total SC remains almost constant at $\sim 8$, while the lobes of the SC trend gradually move at higher lags with increasing values of $N_{T X}$. Conversely, when $\theta_{T X}$ is fixed (Figures $2 \mathbf{b}$ and $3 \mathbf{b}$ ), we have a similar trend in all the MLT cases, with a peak at about lag 19, but the total SC decreases as $N_{T X}$ becomes higher. Both behaviors can be observed in Figures $2 a$ and $3 a$, where $N_{T X}$ and $\theta_{T X}$ change at the same time in the different MLT configurations analyzed.

\subsection{Simulated Images with MLT and Short-Lag F-DMAS}

In this section, images generated with the short-lag F-DMAS formulation are analyzed, and the trend of each performance parameter is reported along with the maximum lag, in the MLT configurations considered.

First analyses have been performed on the simulated PSF at the focal depth, by measuring the lateral resolution at $-6 \mathrm{~dB}$; the results are plotted in Figure 4.

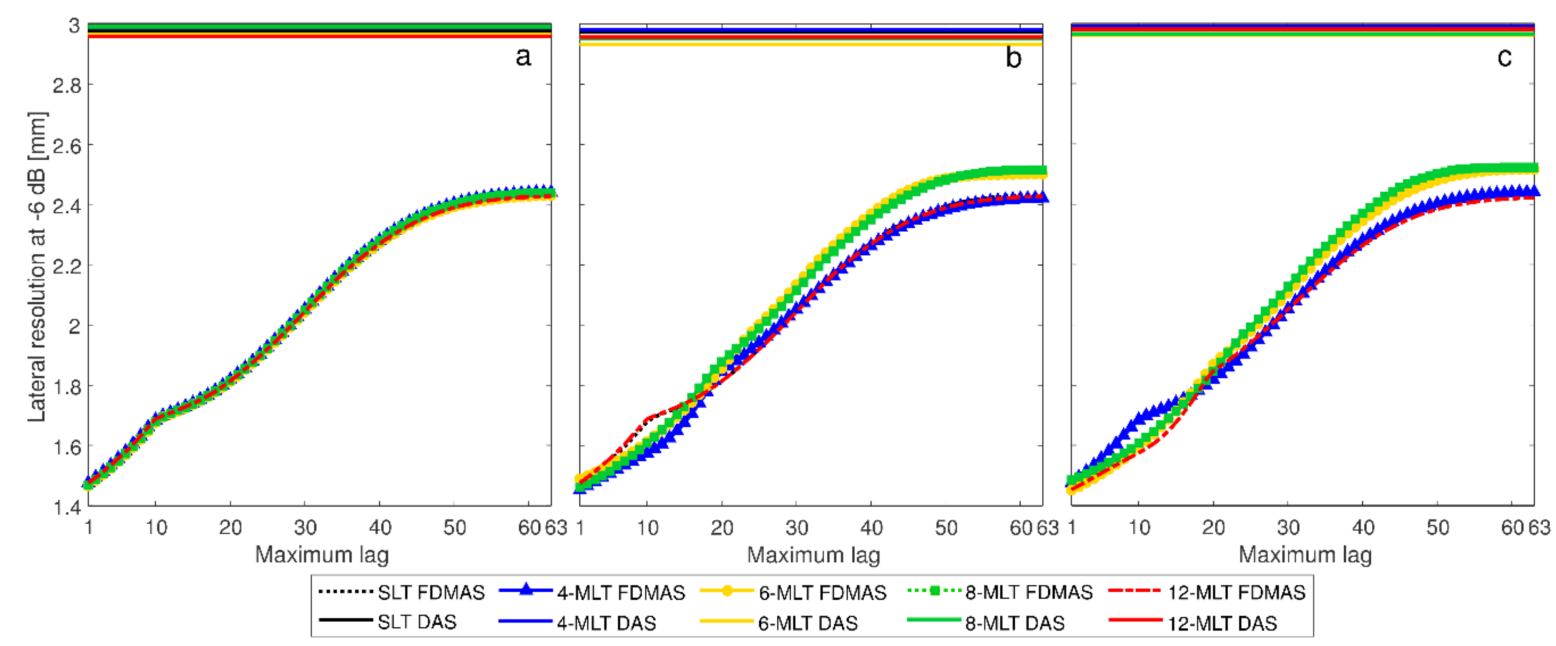

Figure 4. Lateral resolution (at $-6 \mathrm{~dB}$ ) trends, measured on the PSF images with varying maximum-lag, in the different scenarios of Table 1: (a) both the number of TX beams and the angle among them varies (as in SLT or 4/6/8/12-MLT); (b) only the number of TX beams varies $\left(N_{T X}=1 / 4 / 6 / 8 / 12\right)$, while the angular distance among them is fixed to the one of 12-MLT; (c) the number of TX beams is set to four, while the angular distance among them is the one of 4/6/8/12-MLT (in this last case, the legend refers to the number of TX beams which would determine the angular distance). 
The plots in Figure 4a show that no significant difference among lateral resolution curves can generally be observed when the number of TX beams and their angular distance vary, from SLT to 4/6/8/12-MLT, both for DAS and F-DMAS images. In any case, a better resolution is always achieved by F-DMAS, with an increasing trend from $\sim 1.4 \mathrm{~mm}$ at lag 1 to $\sim 2.4 \mathrm{~mm}$ at lag 63 on average, as compared to $\sim 3 \mathrm{~mm}$ on average for DAS.

When the angular distance between multiple TX beams is fixed (Figure $4 \mathrm{~b}$ ), the trend of F-DMAS image resolution remains almost the same, as observed in Figure 4a. The curves are actually slightly different in the 4/6/8/12-MLT cases, but no significant variation is observed: the trend is always increasing and better values are achieved by F-DMAS than DAS for all maximum-lag values.

Similar results are obtained in Figure 4c, when a fixed number (i.e., four) of TX beams is used while varying $\theta_{T X}$.

Therefore, both DAS with Tukey RX apodization and F-DMAS are generally able to keep the lateral resolution unaltered when MLT with an increasing number of TX beams is applied to improve the frame-rate; this is probably because RX cross-talk in MLT mainly affects the lateral sides of the PSFs and not their main lobe width at $-6 \mathrm{~dB}$. However, F-DMAS both reduces RX cross-talk better than DAS and achieves up to a $1.5 \mathrm{~mm}$ better lateral resolution. If we consider the short-lag F-DMAS formulation, lateral resolution varies with the maximum lag employed; in particular, it worsens as the maximum lag increases.

As an example, Figure 5 (which refers to the 4-MLT configuration of scenario 1, cf. Table 1), clearly shows that the standard F-DMAS always achieves better RX cross-talk reduction than DAS, as well as lower side-lobe levels and a higher lateral resolution, which also makes TX cross-talk (that is suppressed in both cases by Tukey TX apodization) be confined to a narrower region [19]. When the maximum lag is reduced to 40 (Figure 5d) or 10 (Figure 5c), the PSF main-lobes become increasingly narrow as compared to the standard F-DMAS case (Figure 5b), but unfortunately, side-lobes and RX cross-talk artifacts become more visible at the same time (which, on the other hand, affects contrast performance, as will be shown hereinafter), becoming even worse than those of DAS when the maximum lag is limited to 10 .
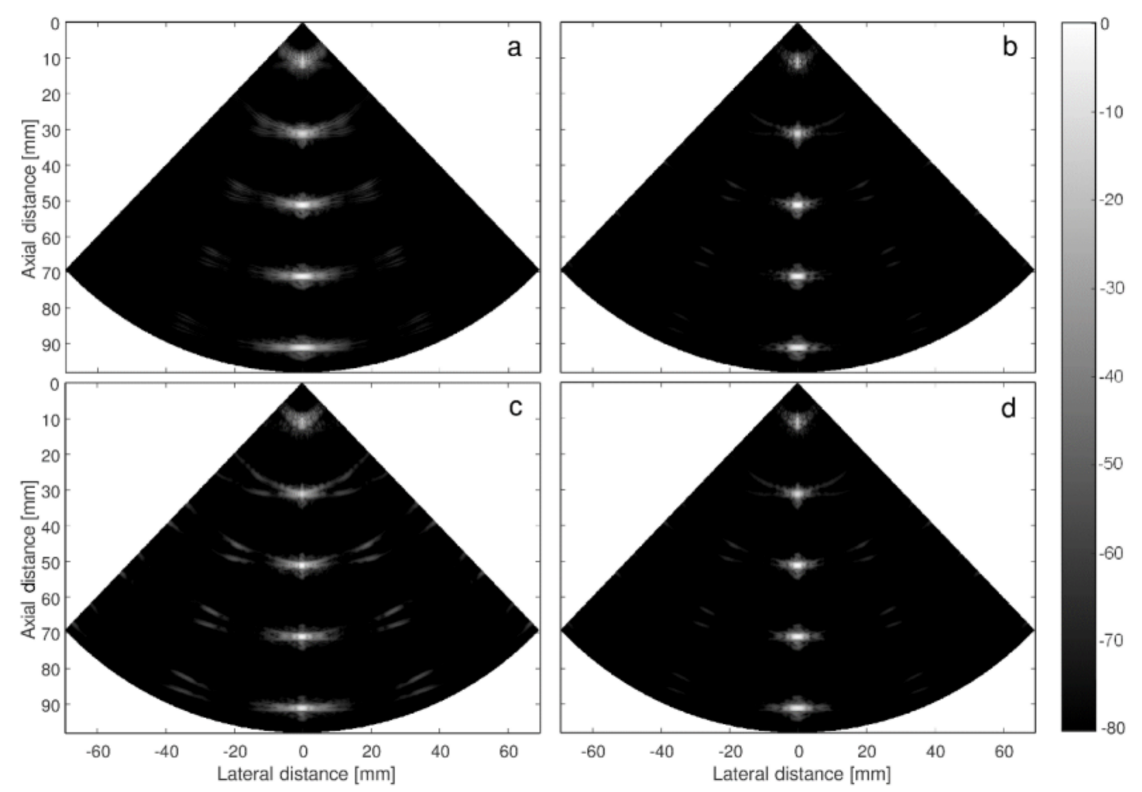

Figure 5. 4-MLT PSF images obtained in scenario 1 by applying (a) DAS with Tukey apodization in TX/RX, or F-DMAS with (b) maximum lag = 63 (standard version), (c) maximum lag = 10, (d) maximum lag $=40$, and Tukey apodization in TX only. Figures are displayed over an $80 \mathrm{~dB}$ dynamic range, in order to better highlight the possible presence of small cross-talk artifacts. 
This can be also observed by looking at Figure 6, which represents the lateral and axial profiles of the PSF at a $70 \mathrm{~mm}$ depth in the same four cases analyzed in Figure 5. In Figure 6a, we can see, for example, that $\mathrm{RX}$ cross talk artifacts (i.e., the two peaks at the left and right sides of the main lobe) are wider and also $15 \mathrm{~dB}$ higher using short-lag F-DMAS with maximum lag $=10$, as compared to the standard F-DMAS case; on the other hand, the main lobe at $-6 \mathrm{~dB}$ is narrower. The four axial sections of the PSFs in Figure $6 \mathrm{~b}$ are instead very similar, also thanks to the application of Tukey TX apodization which lowers TX crosstalk in all cases [22].

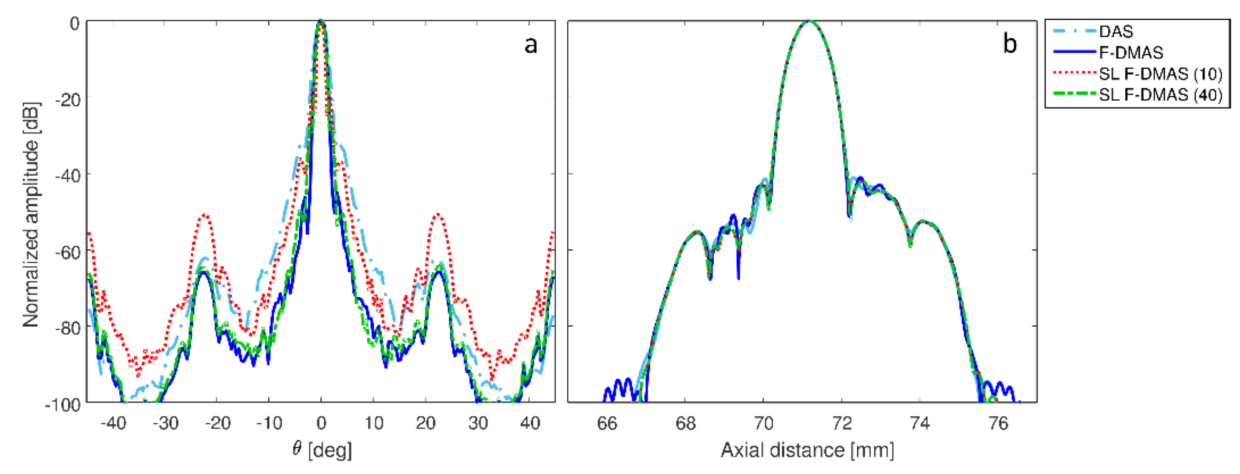

Figure 6. Lateral (a) and axial (b) profiles of the PSF at $70 \mathrm{~mm}$ (i.e., the TX focal depth) shown in Figure 5 for DAS, F-DMAS (standard formulation with maximum lag =63), short-lag F-DMAS with maximum lag $=10$ (SL F-DMAS (10)) and maximum lag = 40 (SL F-DMAS (40)).

The following results show how contrast and speckle quality are influenced by SC variations and by the maximum lag considered in short-lag F-DMAS.

Figure 7 represents the trend of DAS and F-DMAS image CR, CNR, and sSNR over maximum-lag values, measured on the cyst phantom in the three scenarios investigated.

By looking at the curves in Figure 7, we can see at first an increasing trend of the CR (in absolute value) together with the maximum lag employed in F-DMAS. When the classic F-DMAS formulation is used (maximum lag = 63), the CR is always better than that achieved in the corresponding DAS image, for all MLT cases (e.g., 5-8 dB higher in scenario 1). This is not true in the short-lag region, where a maximum-lag threshold value exists, below which the CR of F-DMAS becomes worse than that of DAS. Such a threshold value is variable (generally in a range between lag 29 and 45) and seems to mainly depend on the angular distance between MLT beams (e.g., for 4-MLT in scenario 3 it is equal to 29 or 39 when $\theta_{T X}$ is the one that would be used in 4- or 12-MLT, respectively, cf. Figure 7CR-c). In scenario 2 , this threshold is similar for all plots (i.e., lag $=39 / 41 / 42 / 42$ for $4 / 6 / 8 / 12$ TX beams, cf. Figure 7CR-b), since $\theta_{T X}$ is the same (i.e., $\theta_{12}$ ). Moreover, panels CR-a and CR-c show that there is a dependence of the CR trend on the angular distance among TX beams, but no significant relation exists with the number of MLT beams (in panel CR-b in fact, all curves are almost overlapped). In particular, the CR gets worse as $\theta_{T X}$ decreases from $\theta_{4}$ to $\theta_{12}$.

For what concerns the CNR, the values obtained with F-DMAS are in any case lower than those achieved by DAS, as also previously reported $[8,19]$; their difference becomes higher when MLT is applied, i.e., the CNR at lag 63 with standard F-DMAS in scenario 1 is $\sim 0.4$ and $\sim 0.8$ (on average) lower than with DAS when implementing SLT and MLT, respectively.

The F-DMAS CNR exhibits a different trend compared to CR: it rapidly increases in the short-lag region (up to about lag 10), followed by a plateau up to lag 63 , where the CNR is $\sim 1.3$ on average. Almost the same pattern can be observed in all the three scenarios and for all MLT configurations. By looking at Figure 7CNR-a, CNR-c (scenario 1 and 3), we can also see that the short-lag region trend depends on the angular distance between the multiple TX beams, where lower CNR values are obtained for smaller $\theta_{T X}$ values; on the other hand, when $\theta_{T X}$ is fixed (Figure 7CNR-b, scenario 2), the 
curves are almost overlapped. Thus, the CNR trend also seems to be related to $\theta_{T X}$, even if in a less pronounced way as compared to the CR one.

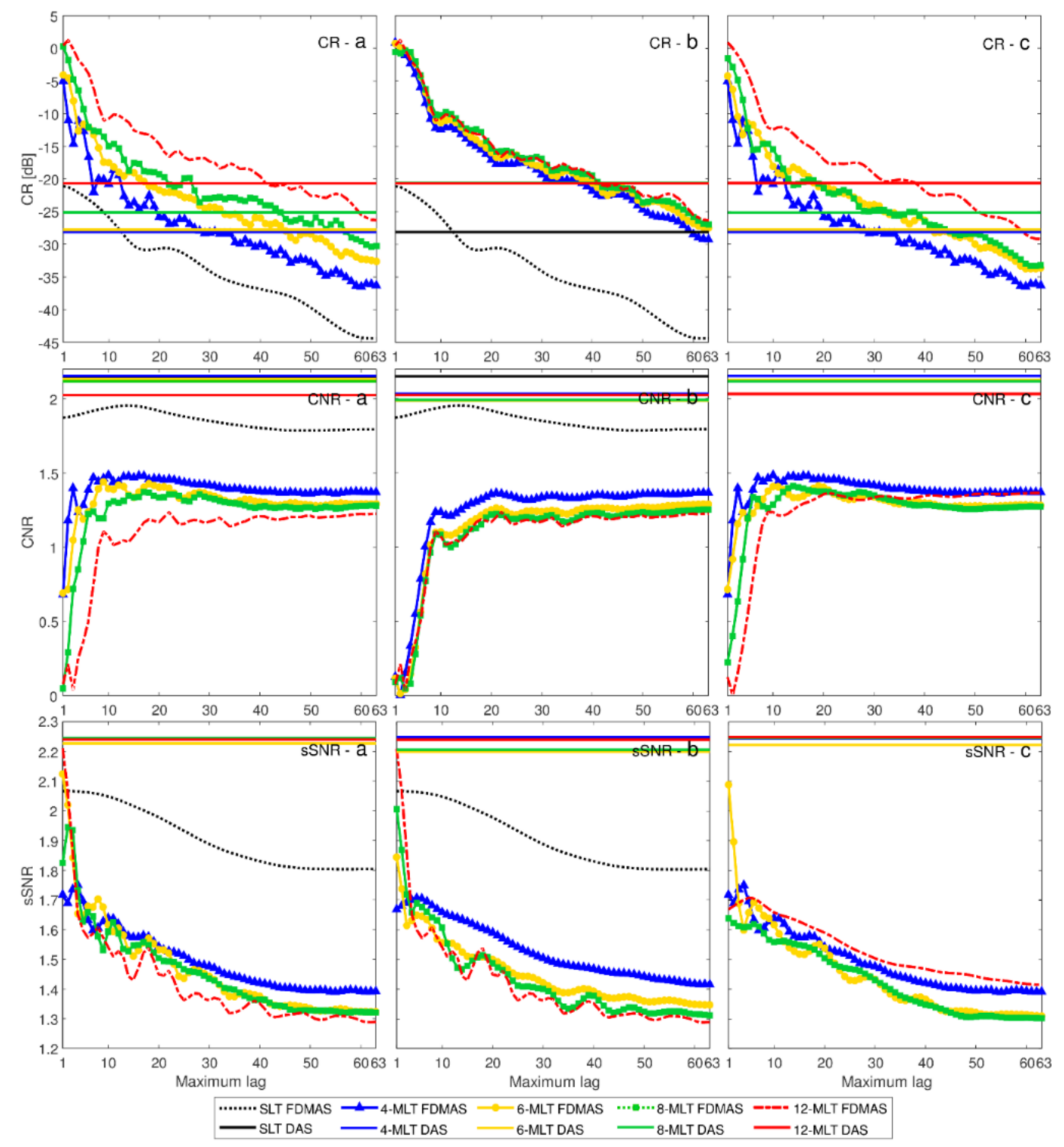

Figure 7. CR (top row), CNR (middle row), and sSNR (bottom row) trends, measured on the cyst-phantom images with varying maximum lag, in the different scenarios of Table 1: (a) both the number of TX beams and the angle among them varies (as in SLT or 4/6/8/12-MLT); (b) only the number of TX beams varies $\left(N_{T X}=1 / 4 / 6 / 8 / 12\right)$, while the angular distance among them is fixed to the one of 12-MLT; (c) the number of TX beams is set to four, while the angular distance among them is the one of 4/6/8/12-MLT (in this last case, the legend refers to the number of TX beams which would determine the angular distance).

Additionally, the F-DMAS sSNR is lower than that achieved by DAS, for all maximum-lag values and in all configurations; this is because F-DMAS impacts the speckle uniformity and a higher variance can be measured, as shown in [8]. As opposed to the CR and CNR trends, the sSNR decreases when the maximum lag increases; however, the total variation is quite small: it settles between 1.3 and 1.7 for lags ranging from 3 to 63, and the highest observable difference between the sSNR at lag 1 and at lag 63 is $\sim 0.9$ for 12-MLT in scenario 1 (Figure 7sSNR-a). Overall, for the sSNR trend, it is hard to 
clearly notice a dependence on the number of TX beams or their angular spacing, as in all scenarios, the curves are very similar.

Finally, to better highlight the short-lag F-DMAS contrast and sSNR performance, an example of images obtained in the classic 4-MLT configuration (i.e., scenario 1, Table 1) is presented in Figure 8. The figure shows an improved image quality achieved by F-DMAS as compared to DAS. Thanks to the higher lateral resolution, the cyst borders are always more clearly visible with F-DMAS, even when the maximum lag is limited to 10 and the CR is lower than that of DAS. The best resolution $(\sim 1.68 \mathrm{~mm})$ is that achieved by the short-lag F-DMAS image with a maximum lag of 10 (Figure 8c); in this case, the cyst dimensions are almost equal to the real ones (i.e., $10 \mathrm{~mm}$ diameter), while for higher lags, it looks elliptic and smaller. When the maximum lag is 40 or 63 (Figure $8 \mathrm{~b}, \mathrm{~d}$ ), the lumen becomes increasingly dark as compared to the short-lag case in Figure $8 c$, and CR increases (about $+9.3 \mathrm{~dB}$ and $+15.5 \mathrm{~dB}$ in absolute value, respectively). This is further highlighted by Figure 9, which compares the cross-sections of the anechoic cysts in Figure 8, showing a definitely lower amplitude level for the pixels in the cyst lumen with standard F-DMAS, as compared to the case with maximum lag $=10$.
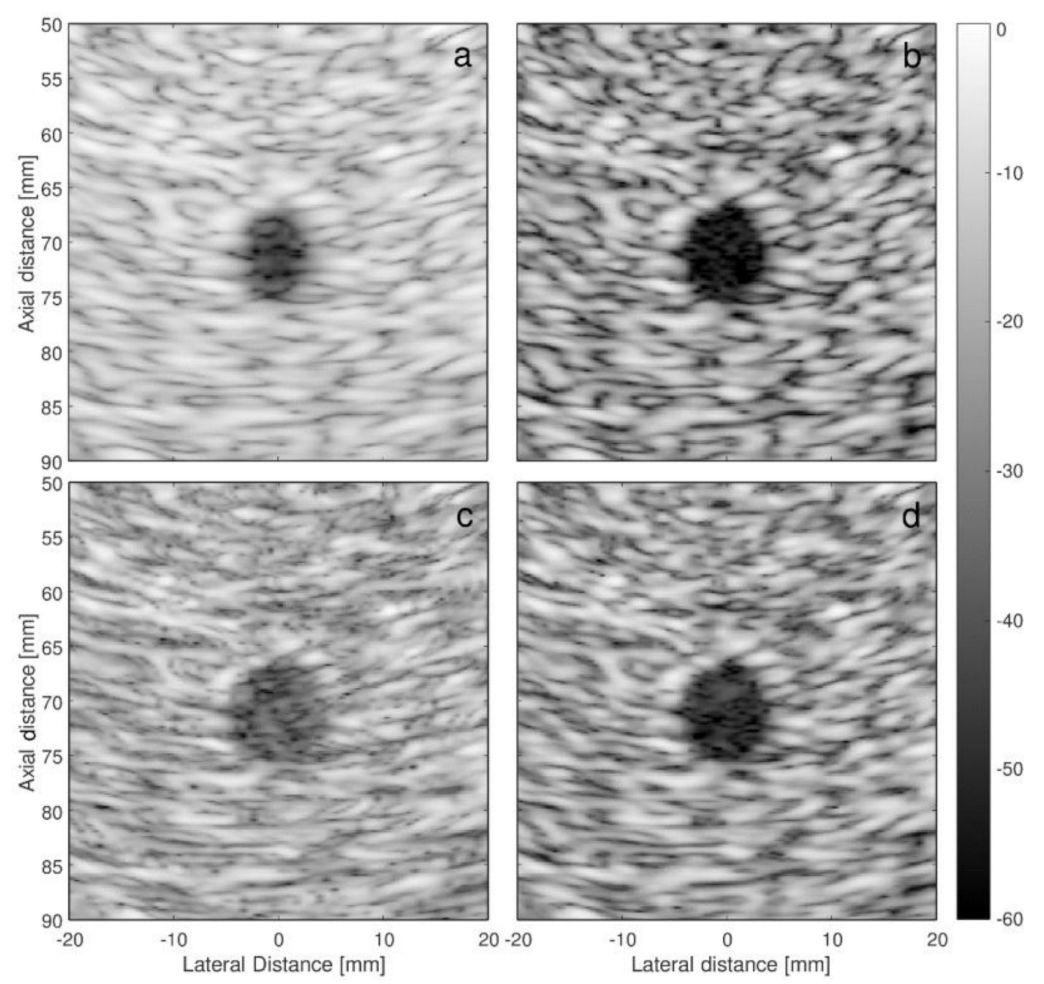

Figure 8. 4-MLT cyst-phantom images obtained in scenario 1 by applying (a) DAS with Tukey apodization in TX/RX, or F-DMAS with (b) maximum lag $=63$ (full-version), (c) maximum lag = 10, (d) maximum lag $=40$, and Tukey apodization in TX only. Figures are displayed over a $60 \mathrm{~dB}$ dynamic range.

The CNR is almost equal in Figure 8 b,d (i.e., $~ 1.4$ ), as well as the sSNR (i.e., $\sim 1.4$ ), and they are $\sim 0.1$ and $\sim 0.25$ lower than in Figure 8c, respectively.

On the whole, the results highlight a dependence of F-DMAS MLT image quality on backscattered signals SC from two points of view.

First, we have shown that, in MLT mode, performance varies with the number and angular spacing of simultaneously transmitted beams, which are in turn related to a variation of backscattered signals SC. The plots highlight that image contrast and speckle uniformity are mainly related to the angular distance between the multiple TX beams, and thus also to the trend of SC over lags; on the contrary, increasing the number of TX beams causes a drop of the measured total SC and has a lower 
impact on these parameters. In general, in fact, $C R$ and CNR decrease when the angle between TX beams reduces, while the sSNR does not show an evident dependency on the number of TX beams or angular spacing. As expected, the lateral resolution measured at $-6 \mathrm{~dB}$ is generally less or not influenced by the implemented MLT configuration.

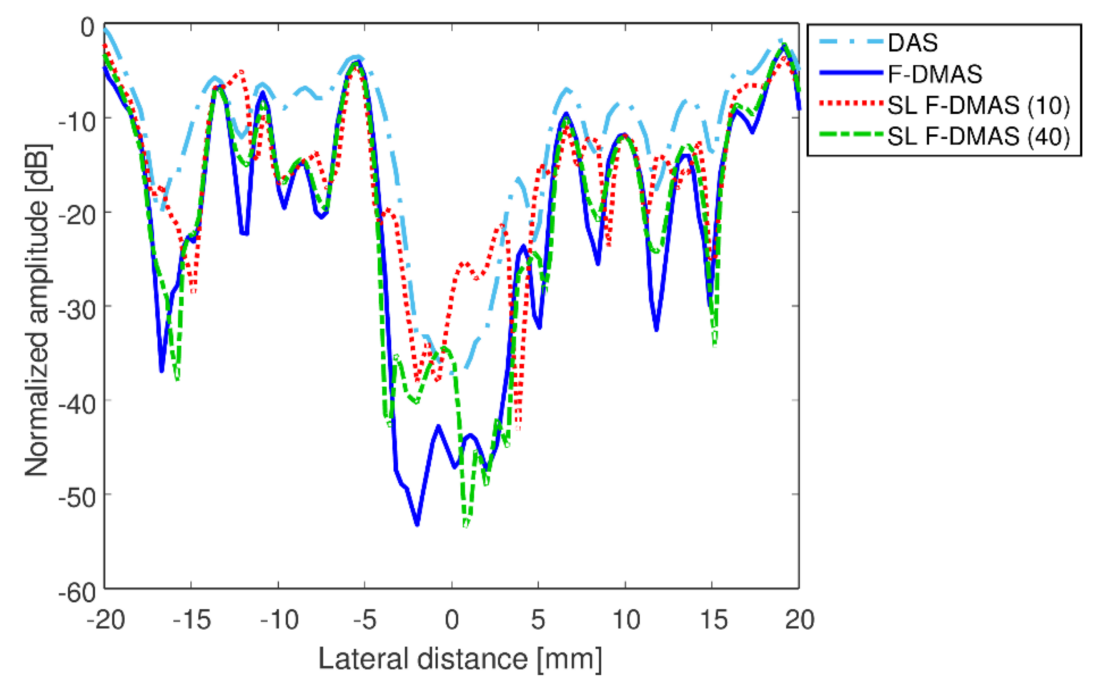

Figure 9. Cross-section of the anechoic cyst in Figure 8, for DAS, F-DMAS (standard formulation with maximum lag = 63), short-lag F-DMAS with maximum lag = 10 (SL F-DMAS (10)) and maximum lag $=40$ (SL F-DMAS (40)).

Secondly, the results demonstrate that a relation exists between the analyzed outcome parameters and the maximum lag of received signals that enter the F-DMAS beamforming algorithm. Particularly, when only short-lag signal couples are used to generate the image, the PSF main lobe is narrower and the resolution is higher, but side-lobe and artifact suppression is not as good as in the full F-DMAS formulation case, which negatively affects image contrast. This is probably because in the short-lag region signals are highly correlated, being recorded by elements separated by a small distance in the probe, but the inclusion of higher-lag components in the F-DMAS summation provides further useful information for a better off-axis artifact rejection.

Albeit the presented analysis does not suggest an absolute criterion for the choice of an optimum maximum-lag value that could maximize all the analyzed performance parameters in F-DMAS images, it provides valuable indications to select a maximum-lag value (and thus a certain trade-off between contrast, resolution, and speckle SNR) based on the specific requirements of the considered application, also relating it to the frame-rate. For example, in those cases in which the contrast achieved by simple DAS could be sufficient but a higher resolution is required, like tissue Doppler applications [28], short-lag F-DMAS with low maximum-lag values can be employed; on the other hand, high maximum-lag values should be used when a high contrast is particularly required (e.g., for an effective segmentation of cardiac walls in ultrasound images [29]).

\section{Conclusions}

In this paper, we have presented an in-depth investigation of the performance of F-DMAS beamforming in the context of MLT imaging; this study is in fact not limited to the classic F-DMAS algorithm case, but it includes a thorough analysis of the image quality achievable with this beamformer when considering different maximum-lag values—and thus using the so called short-lag F-DMAS formulation-in different MLT imaging configurations.

The short-lag F-DMAS formulation provided new insights on the relation between the performance of the F-DMAS algorithm and the SC of backscattered signals. Simulation results show 
that the SC trend over lags and its total value are influenced by the number and angular spacing of simultaneous TX beams. This in turn influences the CNR, CR, sSNR, and resolution of F-DMAS images: higher maximum-lag values improve contrast, while lower values improve resolution and sSNR, which makes the quality of images change with the SC trend.

As a future development of this work, experimental tests will be carried out to assess the impact of noise on the trend of spatial correlation and on the image-quality parameters of interest considered here.

Acknowledgments: This work is supported by the University of Pavia, under the Blue Sky Research project MULTIWAVE. The authors would like to thank Alessandro Savoia (University Roma Tre) for all constructive discussions on the analysis of spatial coherence trends in MLT, and Piero Tortoli (University of Florence) and Giovanni Magenes (University of Pavia) for their insightful comments that helped improve the paper.

Author Contributions: G.M. conceived the study, designed the simulation tests, and worked on the processing of data; A.R. contributed to the design of the study and to simulation tests; G.M. wrote the paper; A.R. revised the manuscript. Both authors read and approved the submitted version of the paper.

Conflicts of Interest: The authors declare no conflict of interest.

\section{References}

1. Mallart, R.; Fink, M. The Van Cittert-Zernike theorem in pulsed ultrasound. Implications for ultrasound imaging. In Proceedings of the IEEE Ultrasonics Symposium, Honolulu, HI, USA, 4-7 December 1990; pp. 1603-1607.

2. Mallart, R.; Fink, M. Adaptive focusing in scattering media through sound-speed inhomogeneities: The Van Cittert Zernike approach and focusing criterion. J. Acoust. Soc. Am. 1994, 96, 3721-3732. [CrossRef]

3. Mallart, R.; Fink, M. The van Cittert-Zernike theorem in pulse echo measurements. J. Acoust. Soc. Am. 1991, 90, 2718-2727. [CrossRef]

4. Liu, D.-L.; Waag, R.C. About the application of the Van Cittert-Zernike theorem in ultrasonic imaging. IEEE Trans. Ultrason. Ferroelectr. Freq. Control 1995, 42, 590-601.

5. Li, P.-C.; Li, M.-L. Adaptive imaging using the generalized coherence factor. IEEE Trans. Ultrason. Ferroelectr. Freq. Control 2003, 50, 128-142. [PubMed]

6. Chen, J.F.; Zagzebski, J.A.; Dong, F.; Madsen, E.L. Estimating the spatial autocorrelation function for ultrasound scatterers in isotropic media. Med. Phys. 1998, 25, 648-655. [CrossRef] [PubMed]

7. Camacho, J.; Parrilla, M.; Fritsch, C. Phase coherence imaging. IEEE Trans. Ultrason. Ferroelectr. Freq. Control 2009, 56, 958-974. [CrossRef] [PubMed]

8. Matrone, G.; Savoia, A.S.; Caliano, G.; Magenes, G. The Delay Multiply and Sum beamforming algorithm in ultrasound B-mode medical imaging. IEEE Trans. Med. Imaging 2015, 34, 940-949. [CrossRef] [PubMed]

9. Lediju, M.A.; Trahey, G.E.; Byram, B.C.; Dahl, J.J. Short-Lag Spatial Coherence of backscattered echoes: imaging characteristics. IEEE Trans. Ultrason. Ferroelectr. Freq. Control 2011, 58, 1377-1388. [CrossRef] [PubMed]

10. Dahl, J.J.; Hyun, D.; Lediju, M.; Trahey, G.E. Lesion detectability in diagnostic ultrasound with short-lag spatial coherence imaging. Ultrason. Imaging 2011, 33, 119-133. [CrossRef] [PubMed]

11. Lu, J.-Y. 2D and 3D high frame-rate imaging with limited diffraction beams. IEEE Trans. Ultrason. Ferroelectr. Freq. Control 1997, 44, 839-856. [CrossRef]

12. Montaldo, G.; Tanter, M.; Bercoff, J.; Benech, N.; Fink, M. Coherent plane-wave compounding for very high frame rate ultrasonography and transient elastography. IEEE Trans. Ultrason. Ferroelectr. Freq. Control 2009, 56, 489-506. [CrossRef] [PubMed]

13. Shattuck, D.P.; Weinshenker, M.D.; Smith, S.W.; von Ramm, O.T. Explososcan: A parallel processing technique for high speed ultrasound imaging with linear phased arrays. J. Acoust. Soc. Am. 1984, 75, 1273-1282. [CrossRef] [PubMed]

14. Mallart, R.; Fink, M. Improved imaging rate through simultaneous transmission of several ultrasound beams. Proc. SPIE 1992, 1773, 120-130.

15. Tong, L.; Ramalli, A.; Jasaityte, R.; Tortoli, P.; D'hooge, J. Multi-transmit beam forming for fast cardiac imaging-Experimental Validation and in vivo application. IEEE. Trans. Med. Imaging 2014, 33, 1205-1219. [CrossRef] [PubMed] 
16. Prieur, F.; Dénarié, B.; Austeng, A.; Torp, H. Multi-line transmission in medical imaging using the second-harmonic signal. IEEE Trans. Ultrason. Ferroelectr. Freq. Control 2013, 60, 2682-2692. [CrossRef] [PubMed]

17. Rabinovich, A.; Feuer, A.; Friedman, Z. Multi-line transmission combined with minimum variance beamforming in medical ultrasound imaging. IEEE. Trans. Ultrason. Ferroelectr. Freq. Control 2015, 62, 814-827. [CrossRef] [PubMed]

18. Zurakhov, G.; Tong, L.; Ramalli, A.; Tortoli, P.; D’hooge, J.; Friedman, Z.; Adam, D. Multi line transmit beamforming combined with adaptive apodization. IEEE Trans. Ultrason. Ferroelectr. Freq. Control 2018. [CrossRef]

19. Matrone, G.; Ramalli, A.; Savoia, A.S.; Tortoli, P.; Magenes, G. High frame-rate, high resolution ultrasound imaging with multi-line transmission and Filtered-Delay Multiply and Sum beamforming. IEEE Trans. Med. Imaging 2017, 36, 478-486. [CrossRef] [PubMed]

20. Matrone, G.; Savoia, A.S.; Caliano, G.; Magenes, G. Depth-of-field enhancement in Filtered-Delay Multiply and Sum beamformed images using synthetic aperture focusing. Ultrasonics 2017, 75, 216-225. [CrossRef] [PubMed]

21. Matrone, G.; Ramalli, A.; Tortoli, P.; Magenes, G. Experimental evaluation of ultrasound higher-order harmonic imaging with Filtered-Delay Multiply And Sum (F-DMAS) non-linear beamforming. Ultrasonics 2018, 86, 59-68. [CrossRef] [PubMed]

22. Tong, L.; Gao, H.; D’hooge, J. Multi-transmit beam forming for fast cardiac imaging-A simulation study. IEEE Trans. Ultrason. Ferroelectr. Freq. Control 2013, 60, 1719-1731. [CrossRef] [PubMed]

23. Bottenus, N.; Byram, B.C.; Dahl, J.J.; Trahey, G.E. Synthetic Aperture Focusing for Short-Lag Spatial Coherence Imaging. IEEE Trans. Ultrason. Ferroelectr. Freq. Control 2013, 60, 1816-1826. [CrossRef] [PubMed]

24. Szabo, T.L. Diagnostic Ultrasound Imaging: Inside Out; Elsevier Academic Press: Hartford, CT, USA, 2004.

25. Lim, H.B.; Nhung, N.T.; Li, E.P.; Thang, N.D. Confocal Microwave Imaging for Breast Cancer Detection: Delay-Multiply-and-Sum Image Reconstruction Algorithm. IEEE Trans. Biomed. Eng. 2008, 55, 1697-1704. [PubMed]

26. Jensen, J.A.; Svendsen, N.B. Calculation of pressure fields from arbitrarily shaped, apodized, and excited ultrasound transducers. IEEE Trans. Ultrason. Ferroelectr. Freq. Control 1992, 39, 262-267. [CrossRef] [PubMed]

27. Jensen, J.A. Field: A program for simulating ultrasound systems. Med. Biol. Eng. Comput. 1996, 34, 351-353.

28. Tong, L.; Ramalli, A.; Tortoli, P.; Fradella, G.; Caciolli, S.; Luo, J.; D’hooge, J. Wide-Angle Tissue Doppler Imaging at High Frame Rate Using Multi-Line Transmit Beamforming: An Experimental Validation In Vivo. IEEE Trans. Med. Imaging 2016, 35, 521-528. [CrossRef] [PubMed]

29. Pedrosa, J.; Queiros, S.; Bernard, O.; Engvall, J.; Edvardsen, T.; Nagel, E.; D’hooge, J. Fast and Fully Automatic Left Ventricular Segmentation and Tracking in Echocardiography Using Shape-Based B-Spline Explicit Active Surfaces. IEEE Trans. Med. Imaging 2017, 36, 2287-2296. [CrossRef] [PubMed]

(c) 2018 by the authors. Licensee MDPI, Basel, Switzerland. This article is an open access article distributed under the terms and conditions of the Creative Commons Attribution (CC BY) license (http://creativecommons.org/licenses/by/4.0/). 